\title{
Survey of the use of therapeutic hypothermia after cardiac arrest in UK paediatric emergency departments
}

\author{
Barnaby R Scholefield, ${ }^{1}$ Mark D Lyttle, ${ }^{2}$ Kathleen Berry, ${ }^{3}$ \\ Heather P Duncan, ${ }^{1}$ Kevin P Morris ${ }^{1}$
}

- Additional material is published online only. To view this file please visit the journa online (http://dx.doi.org/10. 1136/emermed-2011-200348).

${ }^{1}$ Paediatric Intensive Care Unit, Birmingham Children's Hospital, Birmingham, UK

${ }^{2}$ Emergency Department, Royal Children's Hospital, Parkville Victoria, Melbourne, Victoria, Australia

${ }^{3}$ Paediatric Emergency Department, Birmingham Children's Hospital, Birmingham, UK

\section{Correspondence to}

Dr Barnaby R Scholefield, Paediatric Intensive Care Unit, Birmingham Children's Hospital, Steelhouse Lane, Birmingham B4 6NH, UK; barnaby. scholefield@bch.nhs.uk

Accepted 10 January 2012 Published Online First 2 March 2012

\section{ABSTRACT}

Objectives To ascertain current use of therapeutic hypothermia (TH) after paediatric cardiac arrest in UK emergency departments (EDs), and views on participating in a UK randomised controlled trial (RCT) incorporating early induction of $\mathrm{TH}$ in $\mathrm{ED}$.

Design Anonymous web-based survey of 77 UK Emergency Medicine (EM) consultants from 28 UK EDs that see children during the period April-June 2010.

Results $62 \%$ (48/77) of surveyed consultants responded from 21/28 (75\%) EDs. All managed children post cardiac arrest. 90\% (43/48) were aware of the literature concerning $\mathrm{TH}$ after cardiac arrest in adults. However, $63 \%$ (30/48) had never used TH in paediatric practice. All departments had at least one method of inducing $\mathrm{TH}$ (surface cooling; air/water blankets; intravenous cold fluid or catheters). Reasons stated for not inducing $\mathrm{TH}$ included no equipment available (26\%; 11/42), TH not advocated by the local PICU (24\%; 10/42) and not enough evidence for its use (24\%; 10/42). TH was considered based on advice from the local Paediatric Intensive Care Units (68\%; 17/25) or likelihood of recovery after arrest $(32 \% ; 8 / 25)$. There was strong support for a UK RCT of TH versus normothermia (85\%; 40/47). The proposed RCT was felt to be ethical $(87 \% ; 40 / 48)$ with use of deferred consent acceptable (74\%; 34/46).

Conclusion UK EM consultants are aware of TH but infrequently initiate the therapy in children for a number of reasons. Their involvement would enable early induction of $\mathrm{TH}$ in EDs after paediatric cardiac arrest during a UK RCT. The authors have demonstrated the availability of suitable equipment and EM consultant support for participation in such a RCT.

\section{INTRODUCTION}

Therapeutic hypothermia (TH) induced to $33 \pm 1^{\circ} \mathrm{C}$ has been shown to significantly improve neurological outcomes after ventricular fibrillation cardiac arrest in adults and hypoxic-ischaemic encephalopathy in neonates. ${ }^{1-5}$ Whether the same benefit would be achieved after paediatric cardiac arrest is not yet known, although the International Liaison Committee for Resuscitation (ILCOR) recommend "considering the use of $\mathrm{TH}$ for 12-24 hours in infants and children who remain comatose after resuscitation'. 6

Animal studies indicate that there is a narrow therapeutic window for $\mathrm{TH}$, and early treatment appears more efficacious, especially in preventing more severe hypoxic-ischaemic injury after cardiac arrest. $^{7-10}$ Adult and neonatal studies showing neurological benefit have recruited patients to receive $\mathrm{TH}$ within $6 \mathrm{~h}$ of cardiac arrest or hypoxic injury. ${ }^{1-5}$ Whether the same therapeutic window applies to paediatric patients following cardiac arrest is as yet unknown.

Children who suffer out of hospital cardiac arrest are brought to their nearest emergency department (ED) for resuscitation. Following successful resuscitation, these patients are cared for in Paediatric Intensive Care Units (PICUs). With the centralisation of PICUs, these children often require transfer from the presenting ED to a regional PICU, often in a different hospital. ${ }^{11}$ Due to logistical delays in transportation, it may be necessary for $\mathrm{TH}$ to be commenced in the referring ED in order to effectively deliver treatment in the postulated narrow therapeutic window.

The Cold-PACK (Post Arrest Cooling in Kids) study is a multi-centre randomised controlled trial (RCT) comparing $\mathrm{TH}$ with normothermia after paediatric cardiac arrest. It is currently in the protocol design stage, and its feasibility is being assessed in the UK. An important element of design is the ability to recruit patients and initiate treatment early after paediatric cardiac arrest. To achieve this, involvement of Emergency Medicine (EM) consultants working in EDs where children are resuscitated would be essential.

A previous survey of consultants in Paediatric Intensive Care in the UK identified a wide variation in uptake and use of $\mathrm{TH}$ at that time, but demonstrated support for a RCT of TH after paediatric cardiac arrest. ${ }^{12}$ This survey of EM consultants aims to ascertain current practice regarding $\mathrm{TH}$ in UK EDs. In addition, it seeks to explore their attitudes and opinions, and assesses potential support from this group of physicians for the Cold-PACK study.

\section{METHODS}

\section{Survey development}

Relevant questions were generated by the study group and piloted on a group of eight EM consultants from three UK hospitals for further feedback and hyperlink access testing (see online supplementary material).

\section{Survey execution}

Seventy-seven EM consultants from 28 UK EDs were invited to participate. Half were from tertiary children's hospitals which have PICU on-site and see only children. The remainder were from secondary general hospitals which have no PICU on-site and 
see a mix of adults and children. For the purposes of this paper, we use the term EM consultants to refer to all consultants who participated in the survey. An invitation to participate was sent on the 1 April 2010. Invitations were sent three further times and the internet link was closed on the 30 June 2010.

\section{Survey template}

This was created using Microsoft ASP.NET 2008 (Microsoft, Seattle, Washington, USA). Microsoft Excel (Microsoft) was used for data analysis and results are presented as a per cent of survey responders.

\section{RESULTS}

Of 77 EM consultants surveyed, 48 (62\%) responded. Thirty-six $(75 \%)$ were consultants solely in paediatric EM, and $12(25 \%)$ held dual accreditation in adult and paediatric EM. Responses were from 21/28 (75\%) EDs. Ten EDs were located in tertiary children's hospitals which only see children. Eleven were located in secondary general hospitals where consultants manage both adult and paediatric patients.

Consultants responded to questions about their current use of $\mathrm{TH}$ (table 1), how they select patients (table 2), methods of cooling available (table 3 ) and their views on further research (table 4).

\section{Knowledge of the use of TH}

In all, $90 \%(43 / 48)$ of consultants were aware of the literature regarding the use of $\mathrm{TH}$ in adults post cardiac arrest.

Very few used TH after paediatric cardiac arrest; 65\% (30/46) reported use as 'never' and 30\% (14/46) 'seldom'. No responder had a paediatric-specific TH protocol in his or her ED.

A larger proportion used TH after adult cardiac arrest; $27 \%$ (13/48) reported 'always' or 'often'. However, half answered 'not applicable' as they did not manage postcardiac arrest adults. Around $17 \%$ (8/46) reported being aware of an adult $\mathrm{TH}$ protocol in their ED.

The majority (89\%; 42/47) did not know if $\mathrm{TH}$ improved outcome after paediatric cardiac arrest.

\section{Patient selection}

A number of variables were involved when deciding which patients should receive $\mathrm{TH}$. There were also a number of reasons reported by clinicians for not using $\mathrm{TH}$ after cardiac arrest (table $2)$. Around $52 \%(25 / 48)$ responded to the 'reasons stated for selecting patients for TH post cardiac arrest in children'

Table 1 Use of $\mathrm{TH}$

\begin{tabular}{|c|c|c|c|c|c|c|c|}
\hline & & $\mathbf{n}$ & Yes n (\%) & \multicolumn{2}{|c|}{ No n (\%) } & \multicolumn{2}{|c|}{$\begin{array}{l}\text { Unknown } \\
\text { n (\%) }\end{array}$} \\
\hline \multicolumn{2}{|c|}{$\begin{array}{l}\text { Do you look after children post-cardiac } \\
\text { arrest in ED? }\end{array}$} & 48 & $48(100)$ & \multicolumn{2}{|l|}{0} & \multicolumn{2}{|l|}{0} \\
\hline \multicolumn{2}{|c|}{$\begin{array}{l}\text { Are you aware of published research } \\
\text { regarding TH in adults post-cardiac } \\
\text { arrest? }\end{array}$} & 48 & $43(90)$ & \multicolumn{2}{|l|}{$5(10)$} & \multicolumn{2}{|l|}{0} \\
\hline \multicolumn{2}{|c|}{ Do you have a TH protocol for adults? } & 46 & $8(17)$ & \multicolumn{2}{|c|}{$25(52)$} & \multicolumn{2}{|c|}{$13(27)$} \\
\hline \multicolumn{2}{|c|}{ Do you have a TH protocol for children? } & 47 & 0 & \multicolumn{2}{|c|}{$46(98)$} & \multicolumn{2}{|c|}{$1(2)$} \\
\hline \multicolumn{2}{|c|}{$\begin{array}{l}\text { Do you believe TH improves outcome } \\
\text { after paediatric cardiac arrest? }\end{array}$} & 47 & $5(11)$ & 0 & & \multicolumn{2}{|c|}{$42(89)$} \\
\hline & $\mathbf{n}$ & Always & Often & Seldom & Neve & & NA \\
\hline $\begin{array}{l}\text { Do you use TH after adult cardiac } \\
\text { arrest? }\end{array}$ & 48 & $2(4)$ & $11(23)$ & $6(13)$ & $5(10$ & 10) & $24(50)$ \\
\hline $\begin{array}{l}\text { Do you use TH after paediatric } \\
\text { cardiac arrest? }\end{array}$ & 46 & 0 & $2(4)$ & $14(30)$ & 3016 & 65) & 0 \\
\hline
\end{tabular}

ED, emergency department; $\mathrm{TH}$, therapeutic hypothermia.
Table 2 Selection of patients for TH

\begin{tabular}{lr}
\hline $\begin{array}{l}\text { Factors stated in selecting patients for TH post paediatric cardiac } \\
\text { arrest (n=25) }\end{array}$ & $\mathbf{n}(\%)$ \\
\hline On advice from PICU & $17(68)$ \\
Likelihood of patient recovery after the arrest & $8(32)$ \\
Absence of life limiting condition & $6(24)$ \\
Availability of equipment & $6(24)$ \\
\hline Reasons stated for not using TH post paediatric cardiac arrest (n=42) & $\mathbf{n}(\%)$ \\
\hline No equipment available & $11(26)$ \\
Not enough research evidence & $10(24)$ \\
Not advocated by regional PICU & $10(24)$ \\
Not in the advanced paediatric life support guidelines & $9(21)$ \\
Rapid transfer to PICU where TH is usually started & $6(14)$ \\
Technically too difficult & $6(14)$ \\
Not considered for children & $3(7)$ \\
Not an emergency department priority or too infrequent & $3(7)$ \\
The cooling method available is too slow & $2(5)$
\end{tabular}

PICU, Paediatric Intensive Care Unit; TH, therapeutic hypothermia.

question. The most frequent reason was 'on advice from the regional PICU'. Similarly, 24\% (10/42) would not use TH as it was not advocated by the regional PICU and 14\% (6/42) would transfer patients quickly to PICU and felt therapy could be commenced there with minimal delay rather than in the ED. The likelihood of patient recovery was also important for $32 \%$ $(8 / 25)$ in choosing which patients should receive $\mathrm{TH}$.

\section{Methods of inducing hypothermia}

Availability of equipment was reported as a factor in patient selection for $\mathrm{TH}$ and, conversely, lack of equipment was reported as a reason for not using $\mathrm{TH}$ (table 2).

A wide variety of equipment was available in EDs (table 3). ED availability was accepted if any consultant from an ED reported it available. Notably, there were discrepancies between responses from within the same ED regarding available equipment (mean inter-responder agreement score within each ED was 0.37 ; SD 0.06). About $86 \%$ of EDs had access to wet linen and $81 \%$ to ice packs. Cold air circulating blankets were reported to be present in $62 \%(13 / 21)$ of EDs although only $36 \%(15 / 42)$ of individuals reported it being available. There was a median of three methods of cooling (range 1-6) at each ED.

\section{Opinions regarding future research into TH after paediatric cardiac arrest}

A position of clinical equipoise is defined as the existence of an honest, professional disagreement among expert clinicians about the preferred treatment. ${ }^{13}$ Around 52\% (24/46) of EM consultants agreed or strongly agreed that this exists regarding the use of TH after paediatric cardiac arrest. Only 7\% (3/46) 'disagreed' and the remainder were neutral (table 4).

Table 3 Availability of methods for inducing therapeutic hypothermia reported by individuals and per emergency departments

\begin{tabular}{lll}
\hline Methods & $\begin{array}{l}\text { Consultant } \\
\text { response } \\
\mathbf{n}(\%=4)^{*}\end{array}$ & $\begin{array}{l}\text { Emergency department } \\
\text { availability (n=21) } \\
\mathbf{n}(\%)^{*}\end{array}$ \\
\hline Wet linen & $35(83)$ & $18(86)$ \\
Ice-pack to skin & $24(57)$ & $17(81)$ \\
Cold air blanket & $15(36)$ & $13(62)$ \\
Iced cold $\left(4^{\circ} \mathrm{C}\right)$ intravenous saline & $9(21)$ & $5(24)$ \\
Cold water blanket & $2(5)$ & $2(10)$ \\
Intravascular cooling catheter & $1(2)$ & $1(5)$ \\
\hline
\end{tabular}

*Percentage total $>100$ as there was an option to select multiple methods. 
Table 4 Opinions regarding therapeutic hypothermia randomised controlled trial

\begin{tabular}{llllll}
\hline $\begin{array}{l}\text { Please state your strength of } \\
\text { agreement with the following: }\end{array}$ & $\mathbf{n}$ & Agreement* & n (\%) & Mean $\dagger$ & SD \\
\hline There is clinical equipoise & 46 & $24(52)$ & & 3.5 & 0.69 \\
It is ethical to perform a RCT & 46 & $40(87)$ & & 4.2 & 0.72 \\
I approve of the use of deferred consent & 46 & $34(74)$ & & 3.8 & 1.10 \\
\hline Would you: & $\mathbf{n}$ & Yes $\mathbf{n}(\%)$ & No $\mathbf{n}(\%)$ & Don't know n (\%) \\
\hline Support a comparative intervention trial? & 47 & $40(85)$ & $2(4)$ & $5(11)$ \\
Support a method of cooling study? & 45 & $34(76)$ & $5(11)$ & $6(13)$ \\
Allow your patients to be recruited? & 47 & $43(91)$ & $1(2)$ & $3(6)$ \\
Use a trial protocol in your ED? & 48 & $41(85)$ & $1(2)$ & $6(13)$ \\
\hline
\end{tabular}

${ }^{*}$ Agreement $=$ percentage responding 'agree' or 'strongly agree'.

†Mean score on a Likert scale: 5 (strongly agree), 4 (agree), 3 (neutral), 2 (disagree) and 1 (strongly disagree).

Ninety one per cent would agree to their patients being recruited into a RCT of TH after paediatric cardiac arrest. There was greater support for a trial of TH versus normothermia than there was for a method of cooling trial. There was strong support that a RCT of TH is ethical (87\%) and that deferred consent is appropriate (74\%).

\section{DISCUSSION}

The results of this survey suggest that very few UK EM consultants currently initiate $\mathrm{TH}$ after paediatric cardiac arrest in their ED. Various reasons for this emerge from the survey.

EM consultants are unsure whether TH improves outcome after paediatric cardiac arrest. There is an awareness of adult postventricular fibrillation cardiac arrest TH studies which have demonstrated a reduction in bad neurological outcome when treated with TH rather than normothermia. ${ }^{1} 214$ However, the vast majority of responding consultants in this survey did not know if TH improved neurological outcome after paediatric cardiac arrest.

The 2006 ILCOR guideline recommended the consideration of hypothermia therapy after paediatric cardiac arrest. ${ }^{6}$ A more recent edition of the ILCOR guideline has been published since this survey was carried out. However, the recommendations have changed only for adolescents, suggesting that $\mathrm{TH}$ is beneficial after ventricular fibrillation cardiac arrest in this group. ${ }^{15}$ The evidence to support the ILCOR recommendation is from animal, neonatal and the adult studies described above. There has been no paediatric-specific RCT. Only two retrospective observational cohort studies ${ }^{16} 17$ have been published in this area, but they use unbalanced groups and show no difference in outcomes of patients receiving $\mathrm{TH}$ compared with normothermia. This lack of evidence may explain the demonstrated lack of uptake of this recommendation. The ongoing Therapeutic Hypothermia After Paediatric Cardiac Arrest multi-centre RCT (clinical trial.gov ID: NCT00880087 and NCT00878644) in the USA may gather important data on safety and efficacy to strengthen any future guidance.

The role of the regional paediatric intensive care team appears to influence the management of paediatric postcardiac arrest patients in the ED. Of EM consultants who would consider using the therapy, $68 \%$ reported that they would only commence it if their regional PICU requested and advocated it. If the time taken to admit to PICU was anticipated to be short, then therapy would be delayed until then.

A number of methods of cooling appear to be accessible in most EDs. However, between consultants from the same ED, there is discrepancy in the perception of what equipment is available. There was a belief that the PICU would have access to more sophisticated cooling equipment and be able to administer the therapy more safely. This may be due to lack of personal experience and infrequent use of the equipment. Three members of this group have previously reported the results of a survey of UK Paediatric Intensive Care consultants conducted in $2009 .{ }^{12}$ In this survey, 48\% (66/113) of responders 'always' or 'often' used TH after paediatric cardiac arrest. Of those who had used TH, only 30\% (24/73) would actively induce $\mathrm{TH}$ in an $\mathrm{ED}$. The regional variation of paediatric intensive care practice and the low proportion who would consider starting the therapy in the ED mirror the findings in this present study.

\section{Further points}

This survey highlights the lack of existing protocols for the management of paediatric postcardiac arrest temperature. The temperature of paediatric patients transferred to PICU after cardiac arrest tends already to be low. The US study by Fink et al reported a mean postcardiac arrest PICU admission temperature of $34.6^{\circ} \mathrm{C}( \pm 2.2)$. This included all patients admitted irrespective of whether they were subsequently treated with TH or normothermia. ${ }^{16}$ Data for postcardiac arrest temperature of UK children in the ED or PICU are not yet available. However, we hypothesise that young infants and toddlers would generally reduce their core temperature or become frankly hypothermic during or after a cardiac arrest due to the exposure necessary for effective resuscitation despite all efforts to maintain normothermia. Complex methods of inducing $\mathrm{TH}$ may therefore not be required in the initial stages. Larger children and adolescents may require more active therapies to initiate and maintain hypothermia or normothermia. Simple methods such as intravenous iced cold $\left(4^{\circ} \mathrm{C}\right)$ saline have been used successfully in adults ${ }^{18-20}$ and children ${ }^{21} 22$ and should be easily accessible with minimal resource implications. Alternatively, if available, more complex methods such as servocontrolled surface cooling air or water blankets could be used. ${ }^{23}$ However, continuous monitoring of core temperature (rectal, oesophageal or bladder) would be essential in all cases. Further research is needed to enable the development of consensus guidelines on appropriate paediatric temperature monitoring and management. ${ }^{24} 25$

Only $52 \%$ of EM consultants agreed that there is clinical equipoise regarding the use of $\mathrm{TH}$ after paediatric cardiac arrest, although another $41 \%$ were 'neutral'. The presence of clinical equipoise remains a fundamental prerequisite to undertaking a RCT. $^{13}$ There is strong support among EM consultants (85\%) for a comparative trial of TH versus normothermia in paediatric patients post cardiac arrest. A large proportion (91\%) of these consultants would allow their patients to be recruited into such a study. 
The use of deferred consent is still a relatively new process in paediatric resuscitation research in the UK. Changes were made to the Medicines for Human Use (clinical trials) regulations UK in 2008, allowing deferred consent to be used (with ethics committee approval) in emergency resuscitation research. ${ }^{26}$ Approval for the use of deferred consent as demonstrated in this survey $(74 \%)$ would allow very early patient recruitment and initiation of temperature control. This would maximise the potential benefits of $\mathrm{TH}$ by ensuring the desired temperature is reached within the postulated narrow therapeutic window.

\section{Limitations}

This survey targeted EM consultants with direct clinical involvement and decision-making responsibility for paediatric patients. We achieved a credible $62 \%$ response rate, but not all UK EM consultants, some of whom may occasionally manage paediatric cardiac arrest patients, were surveyed. In addition, as with all surveys, the findings only represent self-reported behaviour and do not necessarily equate to actual clinical behaviour. However, the findings of this survey add strength to the case for further investigative research into the use of $\mathrm{TH}$ after paediatric cardiac arrest. We have demonstrated both additional support for, and willingness to participate, in such a RCT, which would incorporate early use of TH in the ED.

\section{CONCLUSION}

This survey of practice and opinions finds that very few UK EM consultants initiate $\mathrm{TH}$ in their EDs after paediatric cardiac arrest. Regional PICUs play significant roles in influencing the management of paediatric cardiac arrest patients. With their support, EM consultants would consider using TH. Although simple methods of cooling patients are already available in most EDs, their use is limited due to clinician concerns regarding safety, lack of protocols and lack of evidence of improved outcomes. There is support among UK paediatric intensive care and EM consultants for further research into the safety and efficacy of $\mathrm{TH}$. This research would require involvement of EM consultants and their departments in order to be able to initiate TH early. This survey supports the proposal that the feasibility of the Cold-PACK Study of $\mathrm{TH}$ versus normothermia commenced in the ED after paediatric cardiac arrest should be explored further.

\begin{abstract}
Acknowledgements We are indebted to the paediatric emergency medicine consultants in the UK who took the time to pilot and complete this survey and we thank Mr David Scholefield for the web-based support and design.
\end{abstract}

Contributors BS and ML designed data collection tools, monitored data collection and analysed the data. BS drafted and revised the paper. BS is guarantor. ML revised the paper. KB, HD and KM piloted data collection tool, analysed the data and revised the paper. DS adapted the data collection tool for internet use.

Competing interests None.

Ethics approval This study was a service evaluation via a survey of medical practitioners working in emergency medicine in the UK; we, therefore, were satisfied that ethics committee approval was not required.

Provenance and peer review Not commissioned; externally peer reviewed.

\section{REFERENCES}

1. Bernard SA, Gray TW, Buist MD, et al. Treatment of comatose survivors of out-of-hospital cardiac arrest with induced hypothermia. N Engl J Med 2002; 346:557-63.

2. HACA. Mild therapeutic hypothermia to improve the neurologic outcome after cardiac arrest. N Engl J Med 2002;346:549-56.

3. Gluckman PD, Wyatt JS, Azzopardi D, et al. Selective head cooling with mild systemic hypothermia after neonatal encephalopathy: multicentre randomised trial. Lancet 2005;365:663-70.

4. Azzopardi DV, Strohm B, Edwards AD, et al. Moderate hypothermia to treat perinatal asphyxial encephalopathy. N Engl J Med 2009;361:1349-58.

5. Shankaran S, Laptook AR, Ehrenkranz RA, et al. Whole-body hypothermia for neonates with hypoxic-ischemic encephalopathy. N Engl J Med 2005;353:1574-84

6. ILCOR. The International Liaison Committee on Resuscitation (ILCOR) consensus on science with treatment recommendations for pediatric and neonatal patients: neonatal resuscitation. Pediatrics 2006;117:e978-88.

7. Colbourne F, Corbett D. Delayed postischemic hypothermia: a six month survival study using behavioral and histological assessments of neuroprotection. J Neurosci 1995:15:7250-60.

8. Markarian GZ, Lee JH, Stein DJ, et al. Mild hypothermia: therapeutic window after experimental cerebral ischemia. Neurosurgery 1996;38:542-50; discussion 51

9. Kuboyama K, Safar P, Radovsky A, et al. Delay in cooling negates the beneficial effect of mild resuscitative cerebral hypothermia after cardiac arrest in dogs: a prospective, randomized study. Crit Care Med 1993;21:1348-58.

10. Iwata 0, Iwata S, Thornton JS, et al. "Therapeutic time window" duration decreases with increasing severity of cerebral hypoxia-ischaemia under normothermia and delayed hypothermia in newborn piglets. Brain Res 2007:1154:173-80.

11. Pearson G, Barry P, Timmins C, et al. Changes in the profile of paediatric intensive care associated with centralisation. Intensive Care Med 2001;27:1670-3

12. Scholefield BR, Duncan HP, Morris KP. Survey of the use of therapeutic hypothermia post cardiac arrest. Arch Dis Child 2010;95:796-9.

13. Freedman B. Equipoise and the ethics of clinical research. $N$ Engl J Med 1987:317:141-5.

14. Arrich J, Holzer M, Herkner H, et al. Hypothermia for neuroprotection in adults afte cardiopulmonary resuscitation. Cochrane Database Syst Rev 2009(4):CD004128.

15. Kleinman ME, de Caen AR, Chameides $L$, et al. Pediatric basic and advanced life support: 2010 International consensus on cardiopulmonary resuscitation and emergency Cardiovascular care science with treatment recommendations. Pediatrics 2010;126:e1261-318.

16. Fink EL, Clark RS, Kochanek PM, et al. A tertiary care center's experience with therapeutic hypothermia after pediatric cardiac arrest. Pediatr Crit Care Med 2010;11:66-74.

17. Doherty DR, Parshuram CS, Gaboury I, et al. Hypothermia therapy after pediatric cardiac arrest. Circulation 2009:119:1492-500.

18. Bernard S, Buist M, Monteiro 0, et al. Induced hypothermia using large volume, ice cold intravenous fluid in comatose survivors of out-of-hospital cardiac arrest: a preliminary report. Resuscitation 2003;56:9-13.

19. Kim F, Olsufka M, Carlbom D, et al. Pilot study of rapid infusion of $2 \mathrm{~L}$ of 4 degrees $C$ normal saline for induction of mild hypothermia in hospitalized, comatose survivors of out-of-hospital cardiac arrest. Circulation 2005;112:715-19.

20. Kim F, Olsufka M, Longstreth WT Jr, et al. Pilot randomized clinical trial of prehospital induction of mild hypothermia in out-of-hospital cardiac arrest patients with a rapid infusion of 4 degrees $C$ normal saline. Circulation 2007;115:3064-70.

21. Fink EL, Kochanek PM, Clark RS, et al. Fever control and application of hypothermia using intravenous cold saline. Pediatr Crit Care Med. Published Online First: 3 November 2010. doi:10.1097/PCC.0b013e3181fe27c7.

22. Kim YM, Jeong JH, Kyong YY, et al. Use of cold intravenous fluid to induce hypothermia in a comatose child after cardiac arrest due to a lightning strike. Resuscitation 2008;79:336-8.

23. Topjian A, Hutchins L, Diliberto MA, et al. Induction and maintenance of therapeutic hypothermia after pediatric cardiac arrest: efficacy of a surface cooling protocol. Pediatr Crit Care Med 2010;12:e127-35.

24. Fink EL, Kochanek PM, Clark RS, et al. How I cool children in neurocritical care. Neurocrit Care 2010;12:414-20.

25. Nunnally ME, Jaeschke R, Bellingan GJ, et al. Targeted temperature management in critical care: a report and recommendations from five professional societies. Crit Care Med 2011;39:1113-25.

26. Department of Health. The medicines for Human use (Clinical trials) and Blood safety and Quality (Amendment) Regulations 2008. Statutory Instrument 2008 No. 941. The Stationery Office limited, 2008. http://www.opsi.gov.uk/si/si2008/pdf/ uksi 20080941 en.pdf (accessed 17 Feb 2009). 